Bulletin of the Institute of Mathematics

Academia Sinica (New Series)

Vol. 13 (2018), No. 4, pp. 397-409

DOI: 10.21915 /BIMAS.2018403

\title{
ON PRIMITIVE AXIAL ALGEBRAS OF JORDAN TYPE
}

\author{
J. I. HALL ${ }^{1, a}$, Y. SEGEV ${ }^{2, b}$ AND S. SHPECTOROV ${ }^{3, c}$ \\ Dedicated to Professor Robert L. Griess, Jr. on the occasion of his 71st birthday

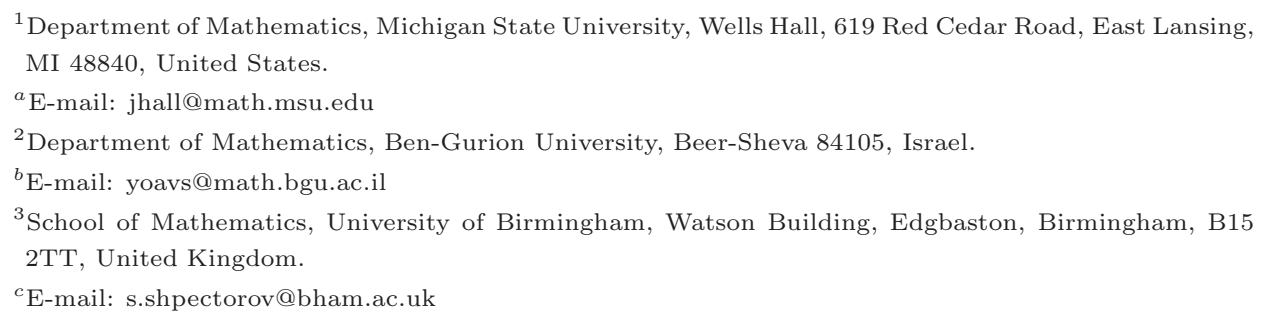

\begin{abstract}
In this note we give an overview of our knowledge regarding primitive axial algebras of Jordan type half and connections between 3-transposition groups and Matsuo algebras. We also show that primitive axial algebras of Jordan type $\eta$ admit a Frobenius form, for any $\eta$.
\end{abstract}

\section{Introduction}

The purpose of this note is threefold. In $\S 2$ we give an overview of our knowledge regarding primitive axial algebras of Jordan type half. This is taken from [2]. In fact we focus in $\S 2$ on one of the main results in [2] which characterizes Jordan algebras of Clifford type amongst primitive axial algebras of Jordan type half. The primitive axial algebras of Jordan type $\eta \neq \frac{1}{2}$ are reviewed (amongst other things) by Jon Hall in another paper of this volume. In $\S 3$, we complete, for the case $\eta=\frac{1}{2}$, a result connecting 3 -transposition groups and Matsuo algebras, established in [1, Theorem 6.3] for $\eta \neq \frac{1}{2}$. In $\S 4$ we show that any primitive axial algebra of Jordan type $\eta$ (any $\eta$ ) admits a Frobenius form.

Received May 9, 2017 and in revised form September 14, 2017.

AMS Subject Classification: Primary: 17A99; Secondary: 17C99, 17B69.

Key words and phrases: Axial algebra, 3-transposition, Jordan algebra, Frobenius form. 
We start by recalling a few definitions. We do not give the historical background as it can be best found in the introduction to [1].

All algebras $A$ in this note are commutative, non-associative over a field $\mathbb{F}$ of characteristic not 2 .

For $a \in A$ the adjoint operator $\operatorname{ad}_{a}$ is multiplication by $a$, so

$$
\operatorname{ad}_{a}: A \rightarrow A, x \mapsto x a
$$

An axis in $A$ is, by definition, a semisimple idempotent, i.e., an idempotent whose minimal ad-polynomial has few distinct linear factors; where the minimal ad-polynomial is the minimal polynomial of the linear operator $\operatorname{ad}_{a}$ (we are not assuming that $A$ is finite dimensional, however, we are assuming that $\operatorname{ad}_{a}$ has a minimal polynomial).

Axial algebras, introduced recently by Hall, Rehren and Shpectorov ([1]), are, by definition, algebras generated by axes. When certain fusion rules, i.e. multiplication rules, between the eigenspaces corresponding to an axis, are imposed the structure of axial algebras remains interesting yet it is more rigid.

Given an element $a \in A$ and a scalar $\lambda \in \mathbb{F}$, the $\lambda$-eigenspace of $\operatorname{ad}_{a}$ is denoted $A_{\lambda}(a)$, so:

$$
A_{\lambda}(a):=\{x \in A \mid x a=\lambda x\} .
$$

(We allow $A_{\lambda}(a)=0$.)

Axial algebras of Jordan type $\eta$, where $\eta \notin\{0,1\}$ is fixed, are algebras generated by a set of axes $\mathcal{A}$ such that for each $a \in \mathcal{A}$ :

(1) The minimal ad-polynomial of $a$ divides $(x-1) x(x-\eta)$.

(2) The fusion rules imitate the Peirce multiplication rules in Jordan algebras. These fusion rules are:

$$
\begin{gathered}
A_{1}(a) A_{1}(a) \subseteq A_{1}(a) \text { and } A_{0}(a) A_{0}(a) \subseteq A_{0}(a), \\
A_{1}(a) A_{0}(a)=\{0\} \\
\left(A_{0}(a)+A_{1}(a)\right) A_{\eta}(a) \subseteq A_{\eta}(a), \quad \text { and } \quad A_{\eta}(a)^{2} \subseteq A_{0}(a)+A_{1}(a) .
\end{gathered}
$$


In particular, if we set

$$
A_{+}(a)=A_{1}(a) \oplus A_{0}(a) \quad \text { and } \quad A_{-}(a)=A_{\eta}(a)
$$

then

$$
A_{\delta}(a) A_{\epsilon}(a) \subseteq A_{\delta \epsilon}(a)
$$

for $\delta, \epsilon \in\{+,-\}$.

Thus, for example, Jordan algebras are axial algebras of Jordan type $\frac{1}{2}$, provided that they are generated by idempotents.

An axis $a \in A$ is absolutely primitive if $A_{1}(a)=\mathbb{F} a$ (this is stronger than the usual notion of primitivity). We call an absolutely primitive axis $a$ satisfying (1), (2) above an $\eta$-axis.

A primitive axial algebra of Jordan type $\eta$ is an algebra generated by $\eta$ axes. For $\eta \neq \frac{1}{2}$, primitive axial algebras of Jordan type $\eta$ were thoroughly analyzed by Hall, Rehren, and Shpectorov in [1]. The case $\eta=\frac{1}{2}$, is much less understood and is of a different nature. This case is the focus of [2] and of $\S \S 2,3$ of this note.

Given an $\eta$-axis $a \in A$, recall that

$$
A=\overbrace{A_{1}(a) \oplus A_{0}(a)}^{A_{+}(a)} \oplus \overbrace{A_{\eta}(a)}^{A_{-}(a)} .
$$

The map $\tau(a): A \rightarrow A$ defined by $x^{\tau(a)}=x_{+}-x_{-}$, where $x=x_{+}+x_{-} \in$ $A_{+}(a)+A_{-}(a)$, is an automorphism of $A$ of order 1 or 2 . It is called the Miyamoto involution corresponding to a.

\subsection{Jordan algebras of Clifford type}

A Jordan algebra of Clifford type $J(V, B)$ consists of the following information:

(1) A vector space $V$ over $\mathbb{F}$ together with a symmetric bilinear form $B$ on $V$. The corresponding quadratic form is denoted $q(v)=B(v, v)$.

(2) The Jordan algebra $J(V, B)$ is $\mathbb{F} \mathbb{1} \oplus V$ with multiplication defined by $\mathbb{1}$ is the identity and $v * w=B(v, w) \mathbb{1}, \quad \forall v, w \in V$. 
The algebra $J(V, B)$ comes from the associative Clifford algebra $\mathrm{Cl}(V, q)$ : it is a sub-Jordan algebra of $\mathrm{Cl}(V, q)^{+}$, where, as usual, $\mathfrak{A}^{+}$denotes the special Jordan algebra that emerges from the associative algebra $\mathfrak{A}$.

Let $J=J(V, B)$. It is easy to check that:

(a) For $u \in V$ and $\alpha \in \mathbb{F}$, the element $\alpha \mathbb{1}+u$ is an idempotent if and only if $\alpha=\frac{1}{2}$ and $q(u)=\frac{1}{4}$.

(b) Assume that $a=\frac{1}{2} \mathbb{1}+u$ is an idempotent in $J$. Then

(i) $J_{1}(a)=\mathbb{F} a$, so $a$ is a $\frac{1}{2}$-axis. (Thus $J(V, B)$ is a primitive axial algebra of Jordan type $\frac{1}{2}$ iff it is generated by idempotents.)

(ii) $J_{0}(a)=\mathbb{F}(\mathbb{1}-a)$ (of course $\mathbb{1}-a$ is a $\frac{1}{2}$-axis), and

(iii) $J_{\frac{1}{2}}(a)=u^{\perp}=J_{\frac{1}{2}}(\mathbb{1}-a)$, where $u^{\perp}=\{v \in V \mid B(u, v)=0\}$.

(c) It follows that $\tau(a)=\tau(\mathbb{1}-a)$, for any $\frac{1}{2}$-axis $a$.

The purpose of $\S 2$ is to show that property (c) above essentially characterizes Jordan algebras of Clifford type amongst primitive axial algebras of Jordan type $\frac{1}{2}$.

\section{Primitive Axial Algebras of Jordan Type Half}

Throughout this section $A$ is a primitive axial algebra of Jordan type $\eta$, generated by a set $\mathcal{A}$ of $\eta$-axes.

Let $\Delta$ be the graph on the set of all $\eta$-axes of $A$, where distinct $a, b$ form an edge iff $a b \neq 0$. Let also $\Delta_{\mathcal{A}}$ be the full subgraph of $\Delta$ on the set $\mathcal{A}$. The purpose of this section is to sketch a proof of the following theorem:

Theorem 2.1. Assume that $\Delta_{\mathcal{A}}$ is connected and that there are two distinct $\eta$-axes $a, b \in A$ such that $\tau(a)=\tau(b)$. Then $\eta=\frac{1}{2}, a+b=\mathbb{1}$ is the identity of $A$, and $A$ is a Jordan algebra of Clifford type.

In the remainder of this section we will sketch a proof of Theorem 2.1. First we need a theorem that enables us to identify $A$ as a Jordan algebra of Clifford type in the case $\eta=\frac{1}{2}$.

Theorem 2.2. Let $\eta=\frac{1}{2}$. Assume that $A$ contains two $\frac{1}{2}$-axes $a, b \in \mathcal{A}$ such that $a+b=\mathbb{1}_{A}$ and such that $v_{a} v_{c} \in \mathbb{F} \mathbb{1}_{A}$, for all $c \in \mathcal{A}$, where $v_{c}=c-\frac{1}{2} \mathbb{1}_{A}$. Then $A$ is a Jordan algebra of Clifford type. 
We do not include a proof of Theorem 2.2, see [2, Theorem 5.4].

We will need some information about 2-generated subalgebras of $A$. This information is taken from [1]. Let $a, b \in \Delta$ with $a \neq b$. We denote by $N_{a, b}$ the subalgebra generated by $a$ and $b$. If $N_{a, b}$ contains an identity element, we denote it by $1_{a, b}$. Note that by [1], 2-generated subalgebras are at most 3-dimensional.

Lemma 2.3 (Lemma 3.1.2 in [2]). Let $a, b \in \Delta$ with $a \neq b$. Then $N_{a, b}$ is 2-dimensional precisely in the following cases:

(1) $a b=0$; we then denote: $N_{a, b}=2 B_{a, b}$.

(2) $\eta=-1, a b=-a-b$; we then denote: $N_{a, b}=3 C(-1)_{a, b}^{\times}$.

(3) $\eta=\frac{1}{2}, a b=\frac{1}{2} a+\frac{1}{2} b$; we then denote: $N_{a, b}=J_{a, b}$.

Furthermore,

(4) the algebras $N_{a, b}$ in cases (2) and (3) above do not have an identity element.

The following proposition deals with 2-generated 3-dimensional subalgebras.

Proposition 2.4 (Proposition $4.6[1]$ ). Let $a, b \in \Delta$ with $a \neq b$. Then $N_{a, b}$ is 3-dimensional precisely when $a b \neq 0$ and there exists $0 \neq \sigma \in N_{a, b}$ and $a$ scalar $\varphi=\varphi_{a, b} \in \mathbb{F}$ such that if we set $\pi=\pi_{a, b}=(1-\eta) \varphi-\eta$, then

(1) $a b=\sigma+\eta a+\eta b$;

(2) $\sigma v=\pi v$, for all $v \in\{a, b, \sigma\}$.

furthermore

(3) $N_{a, b}$ contains an identity element if and only if $\pi \neq 0$, in which case $1_{a, b}=\frac{1}{\pi} \sigma$.

When $N_{a, b}$ is 3-dimensional we denote: $N_{a, b}=B(\eta, \varphi)_{a, b}$, where $\varphi \in \mathbb{F}$ is the scalar mentioned above.

From now on we assume that $\Delta_{\mathcal{A}}$ is connected. Note that by $[2$, Lemma 6.4], $\Delta_{\mathcal{A}}$ is connected iff $\Delta$ is connected. Further, we assume that $a, b \in \Delta$ are distinct with $\tau(a)=\tau(b)$.

Proposition 2.5 (Proposition 6.5 in [2]). $a b=0$ and 
(1) for any $c \in \Delta \backslash\{a, b\}$ exactly one the following holds:

(i) $a c=b c=0$.

(ii) $\eta=\frac{1}{2}$, and for some $x \in\{a, b\}=\{x, y\}$, we have $N_{x, c}=B\left(\frac{1}{2}, 0\right)_{x, c}$ is 3-dimensional, $N_{y, c}=J_{y, c}$ and $N_{y, c} \subset N_{x, c}$. Further $a+b=1_{x, c}$.

(iii) $\eta=\frac{1}{2}, N_{a, c}=N_{b, c}$ is 3-dimensional and $a+b=1_{a, c}$.

(2) If $d$ is an $\eta$-axis in $A$ such that $\tau(d)=\tau(a)$, then $d \in\{a, b\}$.

Proof sketch. By [2, Lemma 3.2.1], for any $c \in \Delta$, we have $a c=0 \Longleftrightarrow$ $c^{\tau(a)}=c$, and since, by definition, $a^{\tau(b)}=a^{\tau(a)}=a$, we see that $a b=0$.

If $a c=0$, then, as above $b c=0$ (and vice versa), so (i) holds. Hence we may assume that $a c \neq 0 \neq b c$.

If $\eta \neq \frac{1}{2}$, then by [1, Proposition 6.5], and since $\Delta$ is connected, $a=b$, a contradiction. Thus $\eta=\frac{1}{2}$.

Now consider

$$
V:=N_{c, c^{\tau(a)}} \subseteq N_{a, c} \cap N_{b, c}
$$

$V$ is either 2 or 3-dimensional. If $V$ is 3-dimensional, then $N_{a, c}=V=N_{b, c}$, and since $a b=0$, one shows that $a+b=1_{a, c}([2$, Lemma 3.2.5]), so (iii) holds.

So suppose $V$ is 2-dimensional. If both $N_{a, c}$ and $N_{b, c}$ are 2-dimensional, then they both equal to $N_{a, b}=\mathbb{F} a \oplus \mathbb{F} b$. But then $c=a$ or $b$, a contradiction.

Therefore without loss $N_{a, c}$ is 3 -dimensional and $V$ is 2-dimensional. If $V=N_{b, c}$ then (ii) holds: Clearly $N_{b, c} \subset N_{a, c}$ and $a+b=1_{a, c}$, and then a careful analysis of the situation gives (ii).

The case where both $N_{a, c}$ and $N_{b, c}$ are 3-dimensional and $V$ is 2dimensional is the hardest case and some precise work is required to get a contradiction.

Proposition 2.6. $\eta=\frac{1}{2}$ and

(1) $x a \neq 0 \neq x b$, for all $x \in \Delta \backslash\{a, b\}$;

(2) A contains an identity element $\mathbb{1}=a+b$;

(3) for any $x \in \Delta$ such that $N_{a, x}$ is 3-dimensional we have $\mathbb{1}=1_{a, x}$. 
Proof. Let $d($,$) be the distance function on \Delta$. Let

$$
\Delta_{1}(a):=\{x \in \Delta \mid d(a, x)=1\} .
$$

Since $\Delta$ is connected $\Delta_{1}(a) \neq \emptyset$. Also, by Proposition $2.5(1 \mathrm{i}), \Delta_{1}(a)=$ $\Delta_{1}(b)$. Let $c \in \Delta_{1}(a)$. By Proposition $2.5, \eta=\frac{1}{2}$ and after perhaps interchanging $a$ and $b, N_{a, c}$ is 3 -dimensional and $a+b=1_{a, c}$. Set

$$
\mathbb{1}=1_{a, c}=a+b,
$$

then

$$
\mathbb{1} c=c, \text { for all } c \in \Delta_{1}(a) \text {. }
$$

Let $y \in \Delta \backslash \Delta_{1}(a)$ be at distance 2 from $a$ in $\Delta$, and let

$$
x \in \Delta_{1}(a) \cap \Delta_{1}(y) .
$$

Without $\operatorname{loss} N_{a, x}$ is 3 -dimensional and $\mathbb{1}=1_{a, x}$. Now

- $a y=0=b y \Longrightarrow \mathbb{1}^{\tau(y)}=(a+b)^{\tau(y)}=a^{\tau(y)}+b^{\tau(y)}=a+b=\mathbb{1}$.

- $\mathbb{1}^{\tau(x)}=\mathbb{1}$ because $\mathbb{1}=1_{a, x}$.

- $\mathbb{1} y=0$ so $\mathbb{1} y^{\tau(x)}=0$.

- $\mathbb{1} x=x$ so $\mathbb{1} x^{\tau(y)}=x^{\tau(y)}$.

- $W:=\operatorname{Span}\left(\left\{y, y^{\tau(x)}\right\}\right) \cap \operatorname{Span}\left(\left\{x, x^{\tau(y)}\right\}\right) \neq\{0\}$. Indeed, $W$ is the intersection of two 2-dimensional subspaces of $N_{x, y}$ which is of dimension at most 3 .

- $\mathbb{1}$ both annihilates and acts as identity on $W$, a contradiction.

Hence $\Delta_{1}(a)=\Delta \backslash\{a, b\}$ and clearly $d(a, b)=2$ in $\Delta$. But now, as we saw above, $\mathbb{1} c=c$ for all $c \in \Delta$. It follows that $\mathbb{1}$ is the identity of $A$ and (3) holds as well.

We are now in a position to prove Theorem 2.1 .

Proof of Theorem 2.1. We show that the hypotheses of Theorem 2.2 are satisfied. By Proposition 2.6, $\eta=\frac{1}{2}$ and $a+b=\mathbb{1}_{A}$. Let $c \in \Delta$. Then

$$
v_{a} v_{c}=\left(a-\frac{1}{2} \mathbb{1}\right)\left(c-\frac{1}{2} \mathbb{1}\right)=a c-\frac{1}{2} a-\frac{1}{2} c+\frac{1}{4} \mathbb{1}=\sigma_{a, c}+\frac{1}{4} \mathbb{1} .
$$


Clearly $v_{a} v_{c} \in \mathbb{F} \mathbb{1}$ if $c \in\{a, b\}$. Otherwise, by Proposition 2.6(1), $a c \neq 0$. If $N_{a, c}$ is 2-dimensional, then since $a c \neq 0, \sigma_{a, c}=0$, and so $v_{a} v_{c} \in \mathbb{F} \mathbb{1}$. If $N_{a, c}$ is 3 -dimensional, then by Proposition 2.6(3), $\mathbb{1}=1_{a, c}$. Furthermore by [1], $\sigma_{a, c}=\pi_{a, c} 1_{a, c}=\pi_{a, c} \mathbb{1}$, for some $\pi_{a, c} \in \mathbb{F}$, and again $v_{a} v_{c} \in \mathbb{F} \mathbb{1}$.

\section{3-transpositions and Matsuo Algebras}

Recall that a set of axes $\mathcal{A}$ is closed iff $a^{\tau_{b}} \in \mathcal{A}$, for all $a, b \in \mathcal{A}$. In this section $A$ is a primitive axial algebra of Jordan type $\eta$ generated by a closed set of $\eta$-axes $\mathcal{A}$, such that $|\mathcal{A}|>1$.

Let $G$ be a group generated by a normal set of involutions $D$. Recall that $D$ is called a set of 3 -transpositions in $G$ if $|s t| \in\{1,2,3\}$, for all $s, t \in D$. The group $G$ is then called a 3-transposition group.

Let $D$ be a normal set of 3-transpositions in the group $G$ that generates $G$. The Matsuo algebra associated with the pair $(G, D)$, denoted here $M_{\delta}(G, D)$, is defined as follows. As a vector space over $\mathbb{F}$ it has the basis $D$. Multiplication is defined for $x, y \in D$ as follows

$$
x \cdot y= \begin{cases}x, & \text { if } y=x \\ 0, & \text { if }|x y|=2 \\ \delta\left(x+y-x^{y}\right), & \text { if }|x y|=3 .\end{cases}
$$

This is extended by linearity to the entire algebra. (Note that we denote multiplication in $G$ by juxtaposition and in $M_{\delta}(G, D)$ by dot.) By [1, Theorem 6.2], $M_{\delta}(G, D)$ is a primitive axial algebra of Jordan type $2 \delta$.

The purpose of this section is to prove the following Theorem:

Theorem 3.1. Suppose that the graph $\Delta_{\mathcal{A}}$ is connected. Let $D:=\left\{\tau_{a} \mid a \in\right.$ $\mathcal{A}\}$ and $G=\langle D\rangle$. Assume that the map $a \mapsto \tau_{a}$ on $\mathcal{A}$ is injective and that $D$ is a set of 3-transpositions in $G$. Then $A$ is a quotient of the Matsuo algebra $M_{\frac{\eta}{2}}(G, D)$.

Remark 3.2. Theorem 3.1 was proved in $\left[1\right.$, Theorem 6.3] for $\eta \neq \frac{1}{2}$. The proof for $\eta=\frac{1}{2}$ needed a correction, in view of [2]. Note that the summand $\oplus_{i \in I} \mathbb{F}$ does not appear in Theorem 3.1 since we are assuming that $\Delta_{\mathcal{A}}$ is connected. We also mention that for $\eta \neq \frac{1}{2}$, the map on $\mathcal{A}$ defined by $a \mapsto \tau_{a}$ is always injective, by [2, Proposition 6.5], and since $\Delta_{\mathcal{A}}$ is connected. 
We included a proof of Theorem 3.1 for all $\eta$ for completeness.

Lemma 3.3. $a b=\frac{\eta}{2} a+\varphi_{a, b} b-\frac{\eta}{2} a^{\tau_{b}}$, for all $a, b \in \mathcal{A}$.

Proof. Clearly this holds when $a=b$ (since, by definition, $\varphi_{a, a}=1$, and $a^{\tau_{a}}=a$ ), so assume $a \neq b$. Suppose first that $N_{a, b}$ is 2-dimensional. We use [2, Lemma 3.1.2]. If $N_{a, b}=2 B_{a, b}$, then $a b=0, \varphi_{a, b}=0$, and $a^{\tau_{b}}=a$ (see also [2, Lemma 3.2.1]), so the claim holds.

Suppose next that $N_{a, b}=3 C(-1)_{a, b}^{\times}$. Then $\eta=-1, a b=-a-b, \varphi_{a, b}=$ $-\frac{1}{2}$ and $a^{\tau_{b}}=-a-b$ (see also [2, Lemma 3.1.8]), so the claim holds.

Assume that $N_{a, b}=J_{a, b}$. Then $\eta=\frac{1}{2}, a b=\frac{1}{2} a+\frac{1}{2} b, \varphi_{a, b}=1$ and $a^{\tau_{b}}=2 b-a$ (see also [2, Lemma 3.1.9]), so again the claim holds.

We may assume that $N_{a, b}$ is 3 -dimensional. Set $\varphi:=\varphi_{a, b}$. By $\left[2\right.$, Theorem 3.1.3(6)], $a^{\tau(b)}=-\frac{2}{\eta} \sigma-\frac{2(\eta-\varphi)}{\eta} b-a$. Also, $\sigma=a b-\eta a-\eta b$. Hence we get

$$
\begin{aligned}
& \frac{2}{\eta} \sigma=-a-\frac{2(\eta-\varphi)}{\eta} b-a^{\tau_{b}} \\
& \Longleftrightarrow \sigma=-\frac{\eta}{2} a-(\eta-\varphi) b-\frac{\eta}{2} a^{\tau_{b}} \\
& \Longleftrightarrow a b=\frac{\eta}{2} a+\varphi b-\frac{\eta}{2} a^{\tau_{b}} .
\end{aligned}
$$

Corollary 3.4 (See Corollary 1.2 in [1]). A is spanned over $\mathbb{F}$ by $\mathcal{A}$.

Proof. This is immediate from Lemma 3.3 and the definition of a closed set of axes.

Lemma 3.5. Suppose that

$$
\text { the map } a \mapsto \tau_{a} \text { on } \mathcal{A} \text { is injective. }
$$

Let $a, b \in \mathcal{A}$ be distinct. Then

(1) if $\left(\tau_{a} \tau_{b}\right)^{2}=1$, then $a b=0$.

(2) if $\left(\tau_{a} \tau_{b}\right)^{3}=1$, then $\varphi_{a, b}=\frac{\eta}{2}$.

Proof. (1): By [2, Lemmas 3.2.7(2) and 3.1.6(2)] and by (*), $N_{a, b}=2 B_{a, b}$, so (1) holds (see also [2, Lemma 3.1.2(1a)]).

(2): If $\eta \neq \frac{1}{2}$, then (2) follows from [1, Proposition 4.8]. So suppose $\eta=\frac{1}{2}$. By [2, Lemma 3.2.7(1) and Corollary 3.3.2] and by $(*)$, we get $\varphi_{a, b}=\frac{1}{4}$. 
We can now prove Theorem 3.1.

Proof of Theorem 3.1. Set $M:=M_{\frac{\eta}{2}}(G, D)$. We claim that the map

$$
f: M \rightarrow A: \tau_{a} \mapsto a,
$$

extended by linearity is a surjective algebra homomorphism. Note that $f$ is well defined since the map $a \mapsto \tau_{a}$ is injective on $\mathcal{A}$.

Now $f$ is surjective by Corollary 3.4. Next we need to check that

$$
f\left(\tau_{a} \cdot \tau_{b}\right)=a b, \text { for all } a, b \in \mathcal{A} .
$$

If $a=b$, then $\tau_{a} \cdot \tau_{b}=\tau_{a}$, and $a b=a$, so $(*)$ holds.

If $\left|\tau_{a} \tau_{b}\right|=2$, then $\tau_{a} \cdot \tau_{b}=0$, while by Lemma $3.5(1), a b=0$, so $(*)$ holds in this case as well.

Finally assume that $\left|\tau_{a} \tau_{b}\right|=3$. Then

$$
\tau_{a} \cdot \tau_{b}=\frac{\eta}{2}\left(\tau_{a}+\tau_{b}-\tau_{a}^{\tau_{b}}\right)=\frac{\eta}{2}\left(\tau_{a}+\tau_{b}-\tau_{a^{\tau_{b}}}\right),
$$

where the last equality follows from the standard fact that $\tau_{a}^{\tau_{b}}=\tau_{a} \tau_{b}$. Thus $f\left(\tau_{a} \cdot \tau_{b}\right)=\frac{\eta}{2}\left(a+b-a^{\tau_{b}}\right)$. However, by Lemma 3.5(2) and Lemma 3.3, $a b=\frac{\eta}{2}\left(a+b-a^{\tau_{b}}\right)$, so $(*)$ holds in this case as well and the proof of the theorem is complete.

\section{The Existence of a Frobenius Form}

Recall that a non-zero bilinear form $(\cdot, \cdot)$ on an algebra $A$ is called Frobenius if the form associates with the algebra product, that is,

$$
(x y, z)=(x, y z)
$$

for all $x, y, z \in A$.

For primitive axial algebras of Jordan type $\eta$, we specialize the concept of Frobenius form further by asking that the condition $(e, e)=1$ be satisfied for each $\eta$-axis $e$.

The purpose of this section is to prove the following theorem: 
Theorem 4.1. Let $A$ be a primitive axial algebra of Jordan type $\eta$. Then $A$ admits a Frobenius form $(\cdot, \cdot)$. Furthermore, $(e, u)=\varphi_{e}(u)$, for any $\eta$-axis $e \in A$ and any $u \in A$.

The proof of Theorem 4.1 depends on two properties of primitive axial algebras of Jordan type. The first is Corollary 3.4. The second is proven in [1] (Lemma 4.2 below).

For an $\eta$-axis $e \in A$, let $\varphi_{e}$ be the projection function with respect to $e$. That is, for $u \in A$, we have that $u=\varphi_{e}(u) e+u_{0}+u_{\eta}$, where $u_{0}$ and $u_{\eta}$ are eigenvectors of the adjoint linear transformation $\mathrm{ad}_{e}$ for the eigenvalues 0 and $\eta$, respectively.

Lemma 4.2 (Lemma 4.4 in [1]). For a primitive axial algebra $A$ of Jordan type and for any $\eta$-axes $e, f \in A$, we have $\varphi_{e}(f)=\varphi_{f}(e)$.

Note that by [1] the constant $\varphi_{a, b}$, that we used earlier for $\eta$-axes $a, b$, is the same as $\varphi_{a}(b)$.

Proof of Theorem 4.1. We start by defining the bilinear form $(\cdot, \cdot)$ on $A$. Using Corollary 3.4 we can select a basis $\mathcal{B}$ of $A$ consisting of $\eta$-axes, and we let

$$
(a, b)=\varphi_{a}(b), \text { for all } a, b \in \mathcal{B} .
$$

Extending by linearity we get the bilinear form $(\cdot, \cdot)$. Note that Lemma 4.2 implies that $(\cdot, \cdot)$ is symmetric.

Lemma 4.3. (1) $(e, u)=\varphi_{e}(u)$, for all $\eta$-axes $e \in A$ and all $u \in A$;

(2) $(e, e)=1$, for all $\eta$-axes $e \in A$;

(3) $(\cdot, \cdot)$ is invariant under automorphisms of $A$.

Proof. (1\&2): Let $e$ be an $\eta$-axis and suppose that

$$
\varphi_{e}(b)=(e, b), \text { for all } b \in \mathcal{B} \text {. }
$$

Since $\varphi_{e}$ is linear,

$$
\varphi_{e}(u)=\varphi_{e}\left(\sum_{b \in \mathcal{B}} \alpha_{b} b\right)=\sum_{b \in \mathcal{B}} \alpha_{b} \varphi_{e}(b)
$$




$$
=\sum_{b \in \mathcal{B}} \alpha_{b}(e, b)=\left(e, \sum_{b \in \mathcal{B}} \alpha_{b} b\right)=(e, u),
$$

and (1) holds for $e$. Now if $e=a \in \mathcal{B}$, then (*) holds by definition, so (1) holds for $a$. Suppose $e \notin \mathcal{B}$. Let $b \in \mathcal{B}$. Then $\varphi_{e}(b)=\varphi_{b}(e)$, by Lemma 4.2 , and $\varphi_{b}(e)=(b, e)$, as $(1)$ holds for $b$. Finally, since $(\cdot, \cdot)$ is symmetric $(b, e)=(e, b)$, so $\varphi_{e}(b)=(e, b)$, and $(*)$ holds for any $\eta$-axis $e$. This shows that (1) holds.

In particular, for every $\eta$-axis $e \in A$, we have that $(e, e)=1$, since, clearly, $\varphi_{e}(e)=1$. Thus (2) holds.

(3): Let $\psi \in \operatorname{Aut}(A)$, if $u=\varphi_{e}(u) e+u_{0}+u_{\eta}$ is the decomposition of $u \in A$ with respect to the $\eta$-axis $e$, then $u^{\psi}=\varphi_{e}(u) e^{\psi}+u_{0}^{\psi}+u_{\eta}^{\psi}$ is the decomposition of $u^{\psi}$ with respect to the $\eta$-axis $e^{\psi}$. Hence $\varphi_{e^{\psi}}\left(u^{\psi}\right)=\varphi_{e}(u)$, and so $\left(e^{\psi}, u^{\psi}\right)=(e, u)$. Finally, taking an arbitrary $v \in A$ and decomposing it with respect to the basis $\mathcal{B}$ as $v=\sum_{b \in \mathcal{B}} \alpha_{b} b$, we get that $\left(v^{\psi}, u^{\psi}\right)=$ $\left(\sum_{b \in \mathcal{B}} \alpha_{b} b^{\psi}, u^{\psi}\right)=\sum_{b \in \mathcal{B}} \alpha_{b}\left(b^{\psi}, u^{\psi}\right)=\sum_{b \in \mathcal{B}} \alpha_{b}(b, u)=\left(\sum_{b \in \mathcal{B}} \alpha_{b} b, u\right)=$ $(v, u)$. So indeed, $(\cdot, \cdot)$ is invariant under the automorphisms of $A$.

Lemma 4.4. For every $\eta$-axis $e \in A$, different eigenspaces of $\mathrm{ad}_{e}$ are orthogonal with respect to $(\cdot, \cdot)$.

Proof. Clearly, if $u \in A_{0}(e)+A_{\eta}(e)$ then $(e, u)=\varphi_{e}(u)=0$. Hence $A_{1}(e)=$ $\mathbb{F} e$ is orthogonal to both $A_{0}(e)$ and $A_{\eta}(e)$. It remains to show that these two are also orthogonal to each other. Let $u \in A_{0}(e)$ and $v \in A_{\eta}(e)$, the fact that $(\cdot, \cdot)$ is invariant under $\tau_{e}$ gives us $(u, v)=\left(u^{\tau_{e}}, v^{\tau_{e}}\right)=(u,-v)=-(u, v)$. Clearly, this means that $(u, v)=0$.

We are now ready to complete the proof that $(\cdot, \cdot)$ associates with the algebra product. Note that the identity

$$
(x, y z)=(x y, z)
$$

that we need to prove is linear in $x, y$, and $z$. In particular, since $A$ is spanned by $\eta$-axes, we may assume that $y$ is an $\eta$-axis. Furthermore, since $A$ decomposes as the sum of the eigenspaces of $\operatorname{ad}_{y}$, we may assume that $x$ and $z$ are eigenvectors of $\operatorname{ad}_{y}$, say, for the eigenvalues $\mu$ and $\nu$. We have two cases: 
If $\mu=\nu$ then

$$
(x, y z)=(x, \nu z)=\nu(x, z)=\mu(x, z)=(\mu x, z)=(y x, z)=(x y, z)
$$

If $\mu \neq \nu$ then

$$
(x, y z)=\nu(x, z)=0=\mu(x, z)=(x y, z)
$$

since $A_{\mu}(y)$ and $A_{\nu}(y)$ are orthogonal to each other. Thus, in both cases we have the desired equality $(x, y z)=(x y, z)$, proving that the form $(\cdot, \cdot)$ is Frobenius. Also, by Lemma 4.3(1), the second part of Theorem 4.1 holds.

\section{References}

1. J. I. Hall, F. Rehren and S. Shpectorov, Primitive axial algebras of Jordan type, J. Algebra, 437 (2015), 79-115.

2. J. I. Hall, Y. Segev and S. Shpectorov, Miyamoto involutions in axial algebras of Jordan type half, to appear in Israel J. Math. (https://arxiv.org/abs/1610.01307) 